\title{
Sodium Thiosulfate Reduces Acute Kidney Injury in Patients Undergoing Cytoreductive Surgery Plus Hyperthermic Intraperitoneal Chemotherapy with Cisplatin: A Single-Center Observational Study
}

\author{
Annika Kurreck, MD¹, Felix Gronau², Miguel Enrique Alberto Vilchez ${ }^{2}$, Wiltrud Abels, MD $^{3}$, \\ Philipp Enghard, $\mathrm{MD}^{4}$, Andreas Brandl, $\mathrm{MD}^{5}$, Roland Francis, $\mathrm{MD}^{3}$, Bettina Föhre, $\mathrm{MD}^{3}$, \\ Christian Lojewski, $\mathrm{MD}^{2}$, Johann Pratschke, $\mathrm{MD}^{2}$, Peter Thuss-Patience, MD ${ }^{1}$, Dominik Modest, $\mathrm{MD}^{1}$, \\ Beate Rau, $\mathrm{MD}^{2}$, and Linda Feldbrügge, $\mathrm{MD}^{2}$
}

${ }^{1}$ Department of Hematology, Oncology and Tumorimmunology, Charité - Universitätsmedizin Berlin, Corporate Member of Freie Universität Berlin and Humboldt-Universität zu Berlin, Berlin, Germany; ${ }^{2}$ Department of Surgery, Charité Universitätsmedizin Berlin, Corporate Member of Freie Universität Berlin and Humboldt-Universität zu Berlin, Berlin, Germany; ${ }^{3}$ Department of Anesthesiology and Operative Intensive Care Medicine, Charité - Universitätsmedizin Berlin, Corporate Member of Freie Universität Berlin and Humboldt-Universität zu Berlin, Berlin, Germany; ${ }^{4}$ Department of Nephrology and Medical Intensive Care, Charité - Universitätsmedizin Berlin, Corporate Member of Freie Universität Berlin and Humboldt-Universität zu Berlin, Berlin, Germany; ${ }^{5}$ Digestive Unit, Champalimaud Foundation, Lisbon, Portugal

\begin{abstract}
Background. Cytoreductive surgery (CRS) in combination with hyperthermic intraperitoneal chemotherapy (HIPEC) represents a multimodal treatment concept for patients with peritoneal surface malignancies. The use of intraperitoneal cisplatin (CDDP) is associated with a risk of acute kidney injury (AKI). The aim of this study is to evaluate the protective effect of perioperative sodium thiosulfate (STS) administration on kidney function in patients undergoing CRS and CDDP-based HIPEC.

Patients and Methods. We retrospectively analyzed clinical data of all patients who underwent CRS and CDDP-based HIPEC at our hospital between March 2017 and August 2020. Patients were stratified according to the use of sodium thiosulfate (STS vs. no STS). We compared kidney function and clinical outcome parameters between both groups and determined risk factors for postoperative
\end{abstract}

(c) The Author(s) 2021

First Received: 12 March 2021

Accepted: 7 July 2021;

Published Online: 4 August 2021

B. Rau, MD

e-mail: beate.rau@charite.de
AKI on univariate and multivariate analysis. AKI was classified according to acute kidney injury network (AKIN) criteria.

Results. Of 238 patients who underwent CRS and CDDPbased HIPEC, 46 patients received STS and 192 patients did not. There were no significant differences in baseline characteristics. In patients who received STS, a lower incidence $(6.5 \%$ vs. $30.7 \% ; p=0.001)$ and severity of AKI $(p=0.009)$ were observed. On multivariate analysis, the use of STS (OR 0.089, $p=0.001$ ) remained an independent kidney-protective factor, while arterial hypertension (OR 5.283, $p<0.001$ ) and elevated preoperative urea serum level (OR 5.278, $p=0.032$ ) were predictors for postoperative AKI.

Conclusions. The present data suggest that STS protects patients from AKI caused by CRS and CDDP-based HIPEC. Further prospective studies are needed to validate the benefit of STS among kidney-protective strategies.

The efficacy and safety of cytoreductive surgery (CRS) and hyperthermic intraperitoneal chemotherapy (HIPEC) as part of a multimodal therapeutic approach in the adjuvant and palliative setting of peritoneal malignancies is the subject of current research. ${ }^{1}$ 
Cisplatin (CDDP) represents one of the most frequently used intraperitoneal chemotherapeutic agents. CDDP efficacy is enhanced by heat. ${ }^{2}$ Furthermore, higher doses of CDDP can be reached in the peritoneal layer with intraperitoneal compared to systemic administration. ${ }^{3}$ Common adverse effects of CDDP include nausea and vomiting, myelosuppression, polyneuropathy, ototoxicity, and nephrotoxicity. In this context, the incidence of acute kidney injury (AKI) following CDDP-based HIPEC is reported to be up to $20 \%,{ }^{4-8}$ resulting in a prolonged hospital stay and a higher rate of severe morbidities in affected patients. ${ }^{5}$

Sodium thiosulfate (STS) is a water-soluble thiol compound with reducing properties, forming a nontoxic complex with CDDP that is more efficiently eliminated than protein-bound CDDP and was shown to reduce CDDP-induced ototoxicity in children. ${ }^{9,10}$ Intravenously administered STS was found to be highly concentrated in the kidneys. For the purpose of kidney protection, it was first concurrently administered in adult ovarian cancer patients receiving a high-dose systemic chemotherapy with CDDP. $^{11}$

Based on the assumption that STS may also decrease the incidence of AKI in patients receiving intraperitoneal CDDP, it has been used in several clinical HIPEC trials; however, the effects of STS on the incidence and severity of AKI were not examined systematically. ${ }^{12-14}$ Recently, Laplace et al. prospectively evaluated the potential of STS in the prevention of AKI in a small patient cohort undergoing CRS and CDDP-based HIPEC. The authors found STS to significantly reduce the rate of AKI. ${ }^{15}$ To our knowledge, this is the only study evaluating the kidneyprotective potential of STS in patients undergoing CRS and CDDP-based HIPEC by directly comparing with a group of patients not receiving STS. A remaining question is whether severity of postoperative AKI can be influenced, and whether the protective effect of STS can be reproduced in a larger patient cohort.

Based on the aforementioned findings indicating that STS represents a potent kidney-protective drug, we administered STS in all patients receiving CRS and CDDPbased HIPEC in our department starting in November 2019. The aim of the underlying retrospective analysis is to evaluate the kidney-protective potential of STS in a large patient cohort undergoing CRS and CDDP-based HIPEC by comparison with a control group. As this investigation is unplanned and not randomized, the results should be interpreted as hypothesis generating.

\section{PATIENTS AND METHODS}

\section{Patients}

We retrospectively analyzed the clinical data of patients who underwent cytoreductive surgery (CRS) in combination with hyperthermic intraperitoneal chemotherapy (HIPEC) including cisplatin (CDDP) in the Department of Surgery, Campus Charité Mitte and Campus VirchowKlinikum, Charité Universitätsmedizin Berlin, between November 2017 and August 2020.

All patients provided written informed consent to the collection of personal and medical data as well as its use for research purposes, according to the approval by the Charité Institutional Review Board (EA1/009/16). The data collected were stored and processed according to the General Data Protection Regulation and local data protection laws. The retrospective study was conducted in accordance with the ethical standards of the Helsinki Declaration of 1975.

To evaluate the kidney-protective potential of sodium thiosulfate (STS), we compared patients receiving STS with a historical control group of patients not receiving STS and consecutively analyzed both groups with regard to tumor [entity, preoperative therapies, peritoneal cancer index] and patient characteristics [age, sex, comorbidities, physical status according to the American Society of Anesthesiologists (ASA), preoperative renal function] as well as perioperative characteristics [completeness of cytoreduction (CCR), duration of surgery and duration of HIPEC, intraperitoneal chemotherapy and its dose] and postoperative outcome parameters [postoperative renal function, rate of hemodialysis, intensive care unit (ICU) length of stay (LOS) and hospital LOS, postoperative complications].

\section{Definition of Perioperative Characteristics and Postoperative Outcome Parameters}

We evaluated pre- and postoperative renal function by reviewing the following laboratory values prior to the intervention and during the hospital stay until the patient's discharge: serum creatinine $[\mathrm{mg} / \mathrm{dl}]$, estimated glomerular filtration rate $[\mathrm{ml} / \mathrm{min}]$, and serum urea $[\mathrm{mg} / \mathrm{dl}]$. Furthermore, we reviewed medical data for renal replacement therapy following CRS and HIPEC. AKI was defined as a minimum increase of serum creatinine by $0.3 \mathrm{mg} / \mathrm{dl}$ or $150-200 \%$ (1.5- to 2-fold) from baseline value in a 48-h period corresponding to stage 1 based on the classification of the Acute Kidney Injury Network (AKIN). ${ }^{16}$ AKIN stage 2 was defined as an increase of serum creatinine by more than $200-300 \%$ (> 2- to 3-fold). AKIN stage 3 corresponded to an increase of serum creatinine by more than 
$300 \%$ ( $>3$-fold) or a maximum creatinine of at least $4.0 \mathrm{mg} / \mathrm{dl}$ or the need of renal replacement therapy (RRT). ${ }^{16}$ Urine output data were not available; therefore, the definition of AKI was entirely based on serum creatinine.

Peritoneal tumor burden was assessed using the Peritoneal Cancer Index (PCI). ${ }^{17}$ Completeness of cytoreduction was defined according to Sugarbaker et al. as follows: no residual peritoneal lesions $(\mathrm{CCR}=0)$, persisting nodules $<2.5 \mathrm{~mm}$ in size $(\mathrm{CCR}=1)$, nodules between $2.5 \mathrm{~mm}$ and $2.5 \mathrm{~cm}(\mathrm{CCR}=2)$, and nodules $>2.5 \mathrm{~cm}$ or confluent unresectable tumor nodules $(\mathrm{CCR}=3) .{ }^{18}$ The severity of postoperative complications was categorized according to the Clavien-Dindo classification. ${ }^{19}$ ICU LOS was measured from the day of surgery until the patient's release from ICU, and hospital LOS was measured from the day of the intervention until the patient's release from hospital.

For the univariable and the multivariable analysis of potential risk factors for postoperative AKI, we selected cut-offs for the continuous variables of interest as follows: PCI $>15$, age $>60$ years, body mass index $(\mathrm{BMI})>25$ $\mathrm{kg} / \mathrm{m}^{2}$ (definition of overweight and obesity), duration of surgery $>400 \mathrm{~min}$, serum creatinine $>1.0 \mathrm{~m} \mathrm{~g} / \mathrm{dl}$, serum urea $>45 \mathrm{mg} / \mathrm{dl}$, and estimated glomerular filtration rate (eGFR) $<90 \mathrm{ml} / \mathrm{min}$. The cut-offs for the laboratory values were chosen according to the reference values of the laboratory competent for the analysis of our blood samples.

\section{Cytoreductive Surgery (CRS) and Hyperthermic Intraperitoneal Chemotherapy (HIPEC)}

In all of the analyzed patients with peritoneal surface malignancy, the indication for CRS and HIPEC was confirmed by our multidisciplinary tumor board. Patients were preoperatively examined according to the in-house standards for comorbidities that may increase perioperative risk.

Surgery was performed by a small team of specialized surgeons according to our standard operating procedures (SOP) that are developed and regularly revised in a multidisciplinary consortium. In short, following a diagnostic laparoscopy to rule out potential contraindications, a long midline incision and parietal peritonectomy were performed. Further cytoreduction included different organ resections with a substantial variation of the extent of resection. ${ }^{20}$ Subsequent to CRS, inflow and outflow tubes were inserted into the abdomen, and HIPEC was performed after closure of the abdominal wall.

Cisplatin was administered at a dose of $75 \mathrm{mg} / \mathrm{m}^{2}$ of body surface area with either doxorubicin $\left(15 \mathrm{mg} / \mathrm{m}^{2}\right.$ of body surface area) or mitomycin $\mathrm{C}\left(15 \mathrm{mg} / \mathrm{m}^{2}\right.$ of body surface area), using an automatic hyperthermic chemotherapy perfusion device. Chemotherapeutic agents were dissolved in heated saline solution resulting in a total volume of 3-4 1 chemotherapy-containing solution. HIPEC was performed at a flow rate of $400-600 \mathrm{ml} / \mathrm{min}$ for 60 min until December 2018. Starting from January 2019, the total duration of HIPEC was increased to $90 \mathrm{~min}$ according to the protocols used by van Driel et al. and Verwaal et al. ${ }^{12,21}$ The temperature of the chemotherapeutic solution in the abdomen was kept at $43.0 \pm 0.5{ }^{\circ} \mathrm{C}$ and continuously monitored. At the end of perfusion, the remaining fluid was drained without subsequent rinsing.

The postoperative care was strictly governed by our above-mentioned SOP that are guided by current evidencebased recommendations on faster recovery after surgery. This includes restrictive fluid management, optimized pain medication, early mobilization, and early food intake, among other measures.

\section{Administration of Sodium Thiosulfate (STS)}

We administered STS in accordance with the protocol published by van Driel et al. starting in November 2019. ${ }^{12}$ Patients received an STS bolus injection with a dose of $9 \mathrm{~g} /$ $\mathrm{m}^{2}$ of body surface area prior to HIPEC and a continuous application of STS at a dose of $12 \mathrm{~g} / \mathrm{m}^{2}$ of body surface area over $6 \mathrm{~h}$ following HIPEC. Serum sodium concentrations were closely monitored every $1-2 \mathrm{~h}$ for the entire duration of continuous STS administration. The need for dose reduction or discontinuation was consistently reevaluated based on the respective serum sodium concentrations. In the case of serum sodium exceeding $155 \mathrm{mmol} / \mathrm{l}$ following STS bolus injection during HIPEC, continuous administration of STS was not performed. In patients with serum sodium concentrations between 150 and $155 \mathrm{mmol} / \mathrm{l}$, dose of continuous STS administration was reduced by $50 \%$ to a total of $6 \mathrm{~g} / \mathrm{m}^{2}$. In all other cases, full dose of $12 \mathrm{~g} / \mathrm{m}^{2}$ was administered.

\section{Statistical Analysis}

All statistical analyses were performed using SPSS version 25.0 software (IBM Corporation, Armonk, NY, USA). In univariable analysis, categorial variables are presented as numbers (percentages) and were compared using $\chi^{2}$ test; continuous variables are presented as medians (ranges) and were compared using nonparametric MannWhitney U test or analysis of variance (ANOVA). Binary logistic regression was used for multivariate analysis. All variables demonstrating a difference between the analyzed groups $p<0.1$ were included. The two-sided significance level was set to 0.05 with a $95 \%$ confidence interval. 


\section{RESULTS}

\section{Patient and Tumor Characteristics}

We identified 279 adult patients with peritoneal surface malignancies who underwent CRS and HIPEC in our department between November 2017 and August 2020. We excluded 41 patients from analysis who did not receive intraperitoneal CDDP. In the final analysis, 46 patients received STS between November 2019 and August 2020. In 192 patients, CRS and HIPEC were performed without the administration of STS (no STS) between November 2017 and November 2019. Please refer to Fig. 1 for a diagram illustrating the analyzed patient cohort.

The median age of the analyzed patient cohort was 57 years, with a higher percentage of female patients (55.9\% female). The most common tumor entities were gastric cancer followed by low-grade appendiceal mucinous neoplasm (LAMN) and colorectal cancer. Please see Fig. 2 illustrating the distribution of tumor entities within the analyzed patient cohort.

There were no significant differences in baseline characteristics between patients receiving STS and those not receiving STS (no STS). Of note, there were 14 patients (7.3\%) with preexistent renal disease in the no STS group as compared with no patient in the STS group (not significant). Please refer to Table 1 for detailed information on baseline characteristics of all patients and patients separated according to the administration of STS.

\section{Intraoperative Characteristics}

There was a significant difference in the duration of HIPEC between the STS and the no STS group due to the aforementioned change of protocol in January 2019, with prolonged HIPEC duration in the STS group. Apart from this, there were no significant differences between both groups regarding completeness of cytoreduction (CCR),

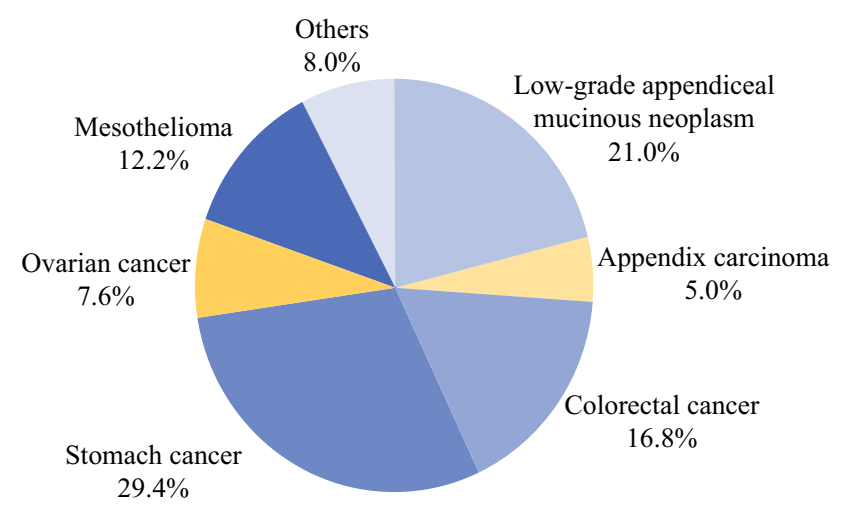

FIG. 2. Distribution of tumor entities within the analyzed patient cohort

duration of surgery, and the combined chemotherapeutic agent during HIPEC. Please refer to Table 1 for detailed information on intraoperative characteristics.

STS caused mild hypernatremia. There was a median increase in serum sodium of $5 \mathrm{mmol} / \mathrm{l}$ (range $1-12 \mathrm{mmol} / \mathrm{l}$ ) following the STS bolus. In patients who received continuous STS in the intended dose, further median increase in serum sodium level was $3.0 \mathrm{mmol} / \mathrm{l}$ (range $1-6 \mathrm{mmol} / \mathrm{l}$ ). Ten patients (21.7\%) either did not receive the continuous administration of STS or received a reduced dose of continuous STS owing to hypernatremia. No patient demonstrated neurological symptoms or other secondary complications of hypernatremia, and none of the patients needed a specific treatment for hypernatremia.

\section{Postoperative Outcome Parameters}

Hospital length of stay (LOS) was comparable between the STS and the no STS group (10 vs. 11 days; $p=0.741$ ), whereas the median ICU LOS was significantly prolonged in the STS as compared with the no STS group (2 vs. 1 day(s); $p=0.031)$.
FIG. 1. Consort diagram of analyzed patient cohort. $C D D P$ cisplatin, STS sodium thiosulfate, $C R$ cytoreductive surgery, HIPEC hyperthermic intraperitoneal chemotherapy

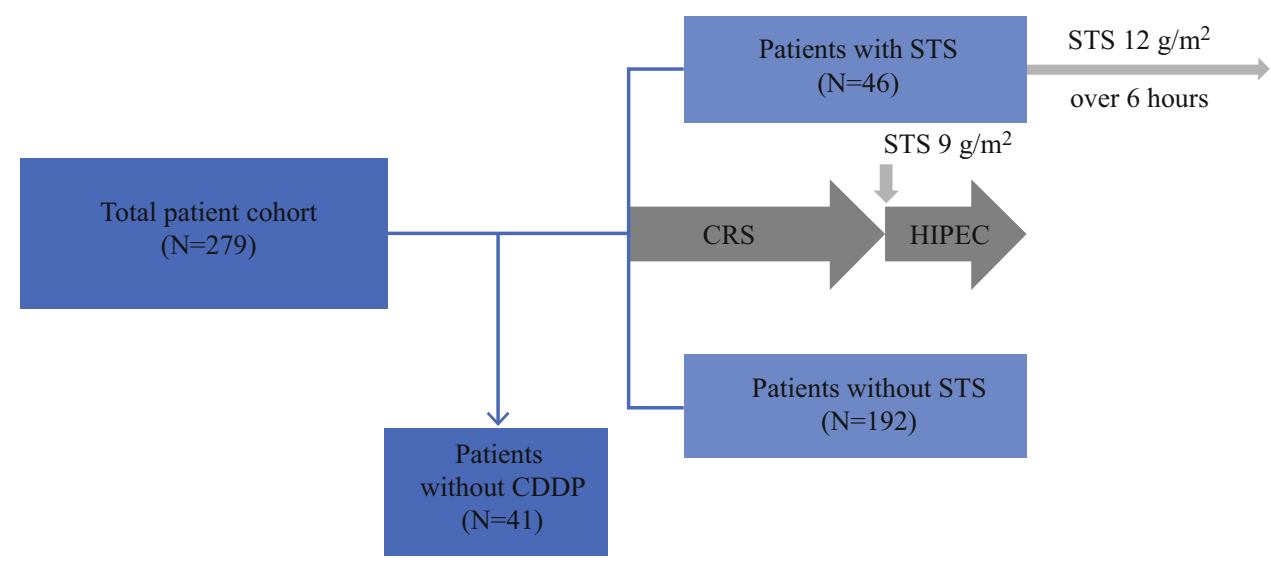


TABLE 1 Baseline and intraoperative characteristics

\begin{tabular}{|c|c|c|c|c|}
\hline & All patients $(N=238)$ & No STS $(N=192)$ & $\operatorname{STS}(N=46)$ & $p$ value \\
\hline Age, median (range) in years & $57(19-83)$ & $57(19-83)$ & $59(23-77)$ & 0.609 \\
\hline Sex, number $(\%)$ & & & & 0.669 \\
\hline Male & $105(44.1)$ & $86(44.8)$ & $19(41.3)$ & \\
\hline Female & $133(55.9)$ & $106(55.2)$ & $27(58.7)$ & \\
\hline Tumor entity, number (\%) & & & & 0.451 \\
\hline Stomach cancer & $70(29.4)$ & $57(29.7)$ & $13(28.3)$ & \\
\hline LAMN & $50(21.0)$ & $42(21.9)$ & $8(17.4)$ & \\
\hline Colorectal cancer & $40(16.8)$ & $29(15.1)$ & $11(23.9)$ & \\
\hline Mesothelioma & $29(12.2)$ & $21(10.9)$ & $8(17.4)$ & \\
\hline Ovarian cancer & $18(7.6)$ & $17(8.9)$ & $1(2.2)$ & \\
\hline Appendix carcinoma & $12(5.0)$ & $10(5.2)$ & $2(4.3)$ & \\
\hline Others & $19(8.0)$ & $16(8.3)$ & $3(6.5)$ & \\
\hline \multicolumn{5}{|l|}{ Comorbidites, number (\%) } \\
\hline Arterial hypertension & $77(32.5)$ & $60(31.4)$ & $17(37.0)$ & 0.471 \\
\hline Diabetes mellitus & $16(6.8)$ & $12(6.3)$ & $4(8.7)$ & 0.558 \\
\hline Coronary artery disease & $13(5.5)$ & $10(5.2)$ & $3(6.5)$ & 0.731 \\
\hline Renal disease & $14(5.9)$ & $14(7.3)$ & $0(0.0)$ & 0.058 \\
\hline Body mass index, median (range) in $\mathrm{kg} / \mathrm{m}^{2}$ & $24.0(14.8-44.8)$ & $24.1(14.8-44.8)$ & $23.6(16.8-44.1)$ & 0.893 \\
\hline Preoperative chemotherapy, number (\%) & $150(63.3)$ & $119(62.3)$ & $31(67.4)$ & 0.520 \\
\hline ASA classification, number $(\%)^{*^{\mathrm{a}}}$ & & & & 0.221 \\
\hline 1 & $11(4.7)$ & $9(4.8)$ & $2(4.3)$ & \\
\hline 2 & $97(41.3)$ & $84(44.4)$ & $13(28.3)$ & \\
\hline 3 & $124(52.8)$ & $94(49.7)$ & $30(65.2)$ & \\
\hline 4 & $3(1.3)$ & $2(1.1)$ & $1(2.2)$ & \\
\hline PCI, median (range) & $12.0(0-39)$ & $11.0(0-39)$ & $12.5(0-39)$ & 0.682 \\
\hline $\mathrm{CCR}$, number $(\%)^{* \mathrm{~b}}$ & & & & 0.174 \\
\hline $0-1$ & $169(77.5)$ & 139 (79.4) & $30(69.8)$ & \\
\hline $2-3$ & $49(22.5)$ & $36(20.6)$ & $13(30.2)$ & \\
\hline Duration surgery, median (range) in minutes & $394(98-765)$ & $389(98-765)$ & $426(162-763)$ & 0.190 \\
\hline Duration HIPEC, median (range) in minutes & $90(30-90)$ & $60(30-90)$ & $90(66-90)$ & $<0.001$ \\
\hline Doxorubicin, number $(\%)^{* \mathrm{c}}$ & $52(21.9)$ & $41(21.4)$ & $11(23.9)$ & 0.706 \\
\hline Mitomycin $\mathrm{C}$, number $(\%)^{* \mathrm{c}}$ & $185(78.1)$ & $150(78.1)$ & $35(76.1)$ & 0.765 \\
\hline
\end{tabular}

The two-sided significance level was set to $p<0.05$

STS sodium thiosulfate, $L A M N$ low-grade appendiceal mucinous neoplasm, ASA physical status according to the classification system of American Society of Anesthesiologists, ${ }^{32}$ PCI peritoneal cancer index, CCR completeness of cytoreductive surgery, ${ }^{18} H I P E C$ hyperthermic intraperitoneal chemotherapy

*a Three patients excluded

${ }^{*}$ Twenty patients excluded

${ }^{* \mathrm{c}}$ One patient excluded because of missing information

There was no statistically significant difference in the number and severity of postoperative complications classified according to Clavien-Dindo. ${ }^{19}$

For detailed information on postoperative outcome parameters please refer to Table 2 .

\section{Preoperative and Postoperative Renal Function}

There were no significant differences in preoperative serum creatinine, serum urea, and glomerular filtration rate between patients in the STS and no STS group.

Postoperatively, the incidence of AKI was significantly lower in the STS group when compared with the no STS group $(6.5 \%$ vs. $30.7 \% ; p=0.001$, Table 2$)$. Within the 
TABLE 2 Postoperative outcome parameters after CRS and HIPEC

\begin{tabular}{|c|c|c|c|c|}
\hline & All patients $(N=238)$ & No STS $(N=192)$ & $\operatorname{STS}(N=46)$ & $p$ value \\
\hline AKIN stage, number (\%) & & & & 0.009 \\
\hline 0 & $176(73.9)$ & $133(69.3)$ & $43(93.5)$ & \\
\hline 1 & $32(13.4)$ & $30(15.6)$ & $2(4.3)$ & \\
\hline 2 & $17(7.1)$ & $16(8.3)$ & $1(2.2)$ & \\
\hline 3 & $13(5.5)$ & $13(6.8)$ & $0(0.0)$ & \\
\hline AKI (AKIN $\geq 1)$,number $(\%)$ & $62(26.1)$ & $59(30.7)$ & $3(6.5)$ & 0.001 \\
\hline Hemodialysis, number (\%) & $6(2.5)$ & $6(3.1)$ & $0(0.0)$ & 0.225 \\
\hline LOS hospital, median (range) in days & $10(3-163)$ & $11(3-163)$ & $10(5-62)$ & 0.741 \\
\hline LOS ICU, median (range) in days & $1(0-153)$ & $1(0-153)$ & $2(1-35)$ & $\mathbf{0 . 0 3 1}$ \\
\hline Clavien-Dindo classification, number $(\%)^{*^{\mathrm{a}}}$ & & & & 0.359 \\
\hline 0 & $88(38.3)$ & $73(38.0)$ & $15(32.6)$ & \\
\hline 1 & $26(11.3)$ & $25(13.0)$ & $1(2.2)$ & \\
\hline 2 & $51(22.2)$ & $38(19.8)$ & $13(28.3)$ & \\
\hline $3 a$ & $24(10.4)$ & $20(10.4)$ & 7 (15.2) & \\
\hline $3 b$ & 25 (10.9) & $21(10.9)$ & $6(13.0)$ & \\
\hline $4 a$ & $9(3.9)$ & $9(4.7)$ & $2(4.3)$ & \\
\hline $4 b$ & $7(3.0)$ & $6(3.1)$ & $2(4.3)$ & \\
\hline 5 & 0 & 0 & 0 & \\
\hline
\end{tabular}

The two-sided significance level was set to $p<0.05$

STS sodium thiosulfate, GFR glomerular filtration rate, AKIN stage severity of acute kidney injury classified according to the Acute Kidney Injury Network, ${ }^{16}$ LOS length of stay, ICU intensive care unit, Clavien-Dindo postoperative complications classified according to ClavienDindo $^{19}$

*a Eight patients excluded because of missing information

patients with AKI, severity according to AKIN stages was significantly lower in the STS group. No patient in the STS group required renal replacement therapy following surgery compared with six patients $(3.1 \%)$ in the no-STS group. This numeric difference did not reach statistical significance $(p=0.225)$. Please see Table 2 for detailed information on the postoperative renal function parameters.

Maximum postoperative serum concentration of creatinine was lower in the STS versus the no STS group $(0.87 \mathrm{mg} / \mathrm{dl}$ vs. $0.97 \mathrm{mg} / \mathrm{dl}, p=0.004)$ with a corresponding higher minimum postoperative eGFR $(88 \mathrm{ml} / \mathrm{min}$ vs. $77 \mathrm{ml} / \mathrm{min}, p=0.007)$. In relation to preoperative baseline values, postoperative creatinine was significantly increased, and eGFR decreased in both groups, but the developments were more pronounced in the no STS group. Serum urea was significantly increased postoperatively in our cohort without a significant difference between the STS and the no-STS group at any time point (Fig. 3).

Of note, when excluding patients with preexisting renal disease from the analysis $(N=14)$, the differences in postoperative renal function parameters between both groups remain significant $(p=0.001)$. Similarly, when excluding all patients who had received $60 \mathrm{~min}$ of HIPEC instead of $90 \mathrm{~min}$, the no STS group $(N=78)$ still had a significantly higher rate of AKI than the STS group (35.9\% vs. $6.5 \%, p<0.001)$.

\section{Risk Factors for Postoperative Acute Kidney Injury}

To identify factors influencing postoperative renal function, patients were grouped according to the diagnosis of postoperative AKI defined as AKIN stage $\geq 1$. Univariable analysis of baseline patient and tumor characteristics as well as intraoperative characteristics demonstrated that patients with AKI were significantly more likely to be male, at an advanced age, overweight, and affected by previous illnesses, such as arterial hypertension, diabetes mellitus, and coronary artery disease. Furthermore, preoperative laboratory parameters indicating impaired renal function (elevated serum creatinine and serum urea, reduced eGFR) were associated with a higher risk to develop postoperative AKI. Please refer to Table 3 for univariable analysis of potential risk factors for postoperative AKI.

On multivariate regression analysis including all preoperative and intraoperative factors that were associated with postoperative AKI in univariable analysis $(p<0.1)$, arterial hypertension (OR 5.283, $p<0.001$ ), preoperative 

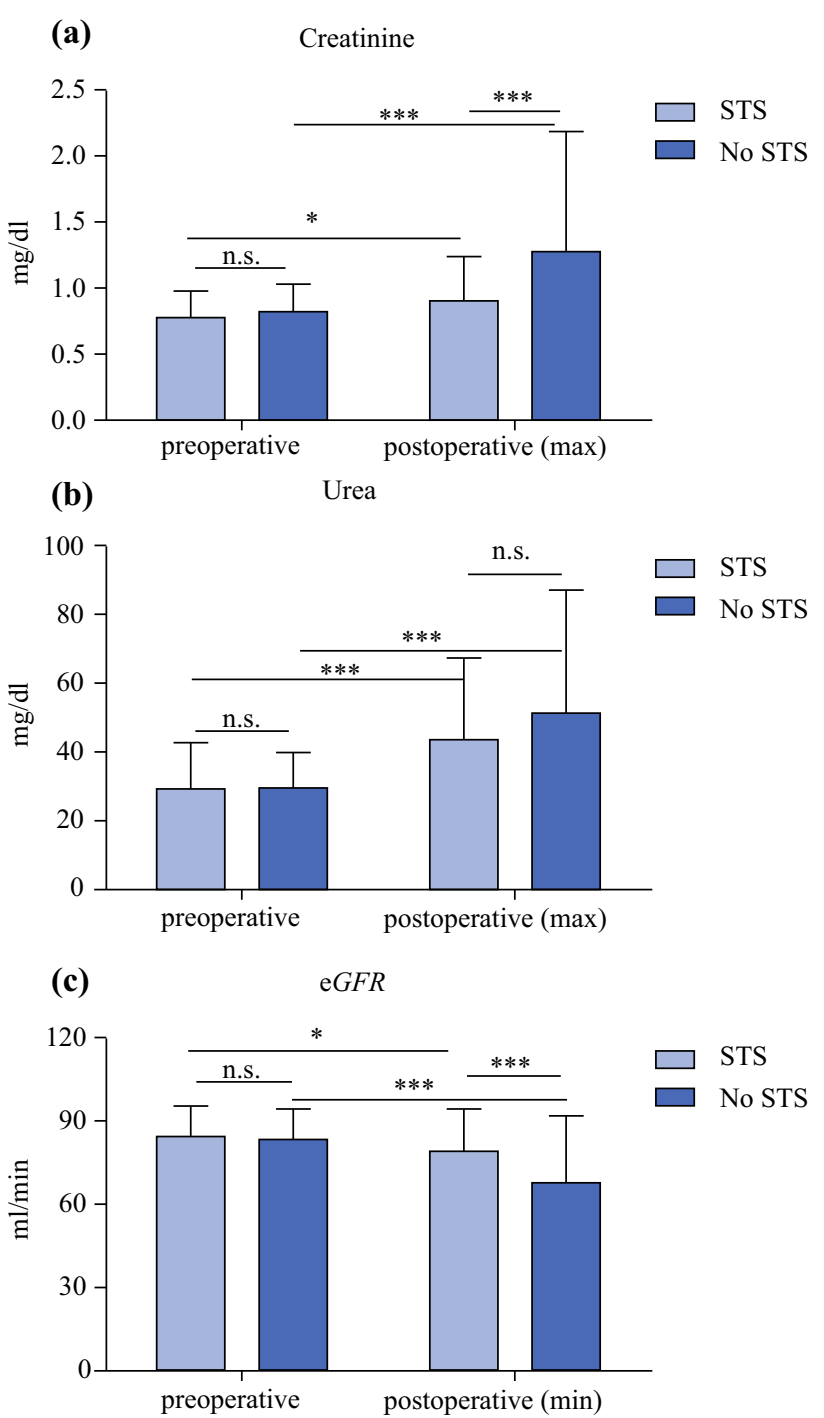

FIG. 3. Laboratory kidney function parameters before and after CRS and HIPEC. Pre- and postoperative serum levels of creatinine (a), urea (b), and estimated glomerular filtration rate (eGFR, c). STS sodium thiosulfate

urea serum level (OR 5.278, $p=0.032$ ), and the use of STS (OR $0.089, p=0.001$ ) remained independent predictive factors (Fig. 4). An extended duration of surgery (longer than $400 \mathrm{~min}$ ) showed a trend towards a higher risk for AKI, but did not reach statistical significance (OR 2.125, $p=0.054)$.

As AKIN stage 1 could be argued to be of low clinical significance, we also performed a multivariate analysis for AKIN stage $\geq 2$ including the same potential risk factors (Supplementary Fig. 1). The results are similar to those of the analysis using AKIN stage $\geq 1$ as a cut-off. The only difference is that preoperative urea serum level in AKIN stages $\geq 2$ no longer represents a significant risk factor for AKI.

\section{DISCUSSION}

The objective of this study was to elucidate to which extent perioperative STS administration protects patients with peritoneal surface malignancy undergoing CRS and CDDP-based HIPEC from postoperative AKI. Additionally, subgroup analyses were performed to identify contributing factors for postoperative renal function.

On analysis, the incidence of AKI in the overall cohort was $26.1 \%$, which is comparable with the rates of AKI following CRS and CDDP-based HIPEC reported in current literature. ${ }^{4-8}$ Patients receiving STS demonstrated a lower incidence and also a lower severity of AKI than those without STS. None of the patients who received STS required renal replacement therapy compared with six patients in the no STS group (n.s.). Furthermore, on multivariate analysis including all factors that were associated with postoperative AKI on univariable analysis, the use of STS remained an independent predictive factor for unimpaired postoperative renal function. Importantly for the clinical relevance of our findings, the results of this multivariate analysis remain significant when using AKIN stage 2 as cut-off for the definition of AKI.

Baseline and intraoperative characteristics including preoperative laboratory renal function parameters were similar between the STS and the no STS group. Due to a change of our HIPEC protocol in January 2019, duration of HIPEC changed from 60 to $90 \mathrm{~min}$, resulting in a significant difference in HIPEC duration in both groups. To control for this potential confounding factor, we performed a subgroup analysis comparing only patients who had received 90 min of HIPEC and obtained the same results with regard to incidence of postoperative AKI.

Our findings are endorsed by the results of a recent study by Laplace et al., who report an AKI incidence of $0 \%$ of STS and $31.4 \%$ of no STS patients $(p<0.05)$. Similar to our results, no patient in the STS-receiving population required renal replacement therapy. ${ }^{15}$ Tilleman et al. administered STS in patients undergoing extrapleural pneumonectomy followed by hyperthermic intrathoracic chemotherapy with CDDP $225 \mathrm{mg} / \mathrm{m}^{2}$ for malignant pleural mesothelioma. ${ }^{22}$ Despite the relatively high CDDP dose administered, AKI of any degree appeared in only $10.8 \%$ of patients. ${ }^{22}$ These findings support the proposal that STS might serve as an effective kidney-protective strategy in patients undergoing CRS and CDDP-based HIPEC.

Apart from the identification of kidney-protective measures, it seems reasonable to search for additional factors influencing AKI in patients treated with CRS and HIPEC. While male gender, advanced age ( $>60$ years), overweight, arterial hypertension, diabetes mellitus, coronary artery disease, and preoperative laboratory parameters 
TABLE 3 Univariable analysis of potential risk factors for postoperative AKI after CRS and HIPEC

\begin{tabular}{|c|c|c|c|}
\hline & AKIN $0(N=176)$ & $\mathrm{AKIN} \geq 1(N=62)$ & $p$ value \\
\hline Advanced age ( $\geq 60$ years) & $61(34.7)$ & $35(56.5)$ & 0.003 \\
\hline Sex, number $(\%)$ & & & 0.048 \\
\hline Male & $71(40.3)$ & $34(54.8)$ & \\
\hline Female & $105(59.7)$ & $28(45.2)$ & \\
\hline Tumor entity, number $(\%)$ & & & 0.835 \\
\hline LAMN & $34(19.3)$ & $16(25.8)$ & \\
\hline Appendix carcinoma & $8(4.5)$ & $4(6.5)$ & \\
\hline Colorectal cancer & $29(16.5)$ & $11(17.7)$ & \\
\hline Stomach cancer & $54(30.7)$ & $16(25.8)$ & \\
\hline Ovarian cancer & $13(7.4)$ & $5(8.1)$ & \\
\hline Mesothelioma & $22(12.5)$ & $7(11.3)$ & \\
\hline Others & $16(9.1)$ & $3(4.8)$ & \\
\hline \multicolumn{4}{|l|}{ Comorbidities, number $(\%)$} \\
\hline Arterial hypertension & $40(22.9)$ & $37(59.7)$ & $<\mathbf{0 . 0 0 1}$ \\
\hline Diabetes mellitus & $8(4.6)$ & $8(12.9)$ & 0.025 \\
\hline Coronary artery disease & $5(2.9)$ & $8(12.9)$ & 0.003 \\
\hline Renal disease & $8(4.6)$ & $6(9.7)$ & 0.143 \\
\hline Overweight (Body mass index $\geq 25 \mathrm{~kg} / \mathrm{m}^{2}$ ) & $70(39.8)$ & $34(54.8)$ & 0.040 \\
\hline Preoperative chemotherapy, number (\%) & $117(66.5)$ & $33(54.1)$ & 0.084 \\
\hline ASA classification, number $(\%)^{*^{\mathrm{a}}}$ & & & 0.232 \\
\hline 1 & $10(5.8)$ & $1(1.6)$ & \\
\hline 2 & $72(41.6)$ & $25(40.3)$ & \\
\hline 3 & $90(52.0)$ & $34(54.8)$ & \\
\hline 4 & $1(0.6)$ & $2(3.2)$ & \\
\hline Advanced peritoneal cancer index $(\mathrm{PCI} \geq 15$ & $66(38.2)$ & $30(50)$ & 0.108 \\
\hline STS, number $(\%)$ & $43(24.4)$ & $3(4.8)$ & 0.001 \\
\hline $\mathrm{CCR}$, number $(\%)^{* \mathrm{~b}}$ & & & 0.945 \\
\hline $0-1$ & $125(77.6)$ & $44(77.2)$ & \\
\hline$>1$ & $36(22.4)$ & $13(22.8)$ & \\
\hline Extended duration of surgery ( $\geq 400 \mathrm{~min}$ ) & 79 (44.9) & $36(58.1)$ & 0.074 \\
\hline Extended duration of HIPEC ( $\geq 60 \mathrm{~min})$ & $93(52.8)$ & $31(50)$ & 0.700 \\
\hline Doxorubicin, number $(\%)^{* \mathrm{c}}$ & $39(22.2)$ & $13(21.0)$ & 0.845 \\
\hline Mitomycin $\mathrm{C}$, number $(\%)^{* \mathrm{c}}$ & $136(77.3)$ & $49(79.0)$ & 0.775 \\
\hline Elevated preoperative creatinine $(\geq 1.0 \mathrm{mg} / \mathrm{dl})$ & $18(10.3)$ & $17(27.4)$ & 0.001 \\
\hline Elevated preoperative urea $(\geq 45 \mathrm{mg} / \mathrm{dl})$ & $4(2.3)$ & $10(17.2)$ & $<\mathbf{0 . 0 0 1}$ \\
\hline Reduced preoperative eGFR $(<90 \mathrm{ml} / \mathrm{min})$ & $60(34.3)$ & $36(58.1)$ & 0.001 \\
\hline
\end{tabular}

The two-sided significance level was set to $p<0.05$

STS sodium thiosulfate, LAMN low-grade appendiceal mucinous neoplasm, ASA physical status according to the classification system of American Society of Anesthesiologists, ${ }^{32}$ PCI peritoneal cancer index, CCR completeness of cytoreduction, ${ }^{18}$ HIPEC hyperthermic intraperitoneal chemotherapy, LOS length of stay, ICU intensive care unit, Clavien-Dindo postoperative complications classified according to ClavienDindo, ${ }^{19}$ GFR glomerular filtration rate

*a Three patients excluded

${ }^{*}$ Twenty patients excluded

${ }^{*}$ One patient excluded

* Eight patients excluded because of missing information

indicating impaired renal function were significantly associated with postoperative AKI on univariable analysis, only arterial hypertension remained an independent risk factor on multivariable analysis. These results are partly consistent with those of an observational study identifying preexisting comorbidities classified according to the 


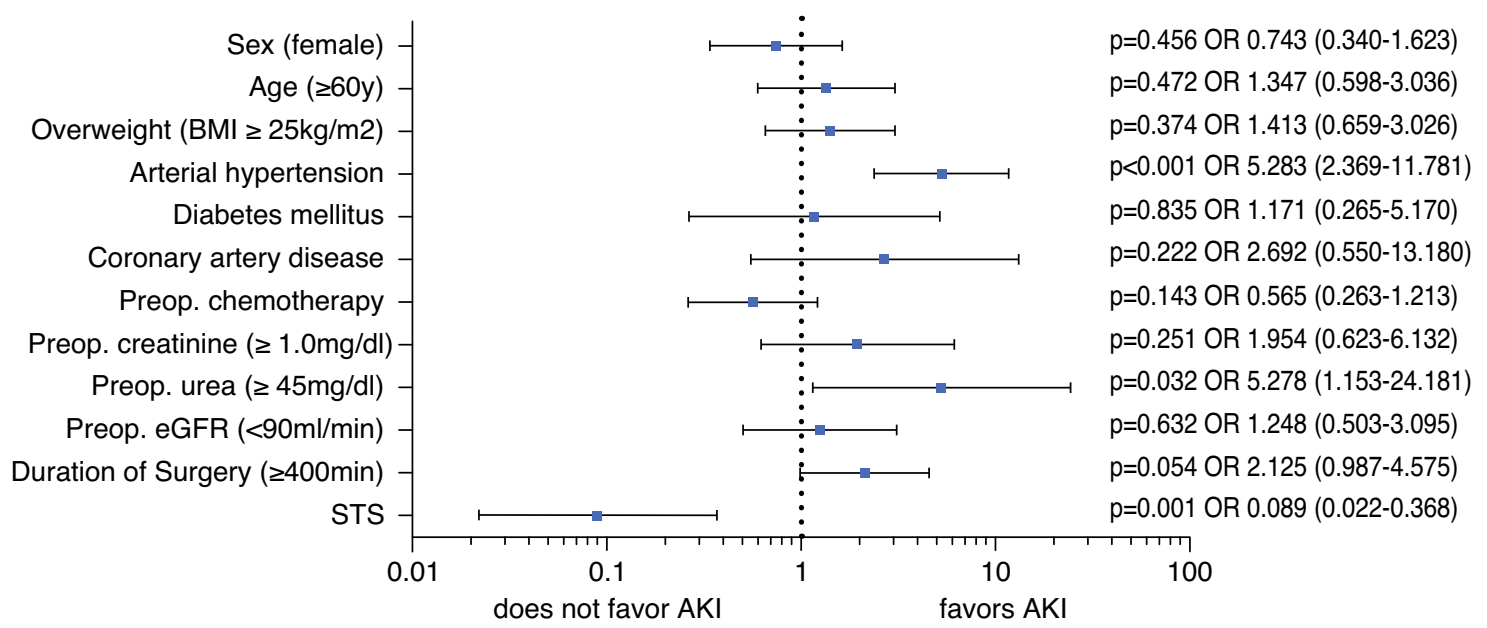

FIG. 4. Multivariate analysis of potential risk factors for postoperative acute kidney injury (AKI) following CRS and HIPEC. OR odds ratio (95\% confidence interval), BMI body mass index, Preop. preoperative, eGFR estimated glomerular filtration rate

Charlson Comorbidity Index as factors significantly associated with AKI following CRS and HIPEC. ${ }^{5}$ In another retrospective study including 475 patients, the authors identified not only advanced age and obesity, but also male gender as risk factors for the development of AKI following CRS and HIPEC. ${ }^{4}$ These findings indicate that there might be a relevant percentage of preexisting, nondiagnosed renal damage in patients with the aforementioned baseline characteristics and comorbidities. It can be concluded that these patients represent a high-risk population with the need for effective kidney-protective strategies and a close monitoring of renal function parameters following CRS and HIPEC.

Apart from patient-specific factors, treatment strategies beyond surgery, especially perioperative fluid management, might have an impact on the incidence of postoperative AKI. In recent years, standards for perioperative care, such as enhanced recovery after surgery (ERAS) guidelines, have been promoted to reduce the length of hospital stay, costs, and complication rates. ${ }^{23,24}$ First published studies in CRS and HIPEC patients show some promising results; however, there was no impact on AKI rates. ${ }^{25-27}$ In our own cohort, fluid management was guided by our SOP for all patients (STS or no STS) and was restrictive after the day of surgery.

On analysis, neither hospital length of stay nor postoperative complication rates differed significantly between patients receiving STS and those not receiving STS, which might be due to the small number of patients developing severe postoperative complications following CRS and HIPEC. The same observation was made by Laplace et al. ${ }^{15}$ The authors did not identify significant differences in the rates of surgical and medical complications other than nephrotoxicity between patients with or without STS administration. However, patients in the STS group stayed a median of two nights in the intensive care compared with one night in the no STS group. Due to the experimental administration of STS based on insufficient clinical evidence in this patient cohort, we exercised caution when hypernatremia as a well-known side effect of STS occurred, resulting in a prolonged ICU stay for the purpose of a close monitoring of electrolyte imbalance until a normalization of serum sodium concentration. This is underlined by the fact that we neither observed complications from hypernatremia nor any specific treatment was required for hypernatremia in our patients.

The occurrence of AKI in our cohort was significantly associated with a prolonged hospital stay. This is in accordance with the results of a previous study reporting on secondary complications caused by renal injury following CRS and HIPEC. ${ }^{5}$ These data underline the importance of avoiding postoperative AKI and consequently reducing the risk for the development of chronic kidney disease with the corresponding implications for subsequent antitumor treatment.

In our study, we observed no side effects from the administration of STS apart from mild hypernatremia. This is in line with previous studies reporting that side effects of STS in humans are rare and mainly grade 1 or 2 according to Common Terminology Criteria for Adverse Events. ${ }^{9,10,12,15}$

In preclinical animal models, STS was not shown to affect antitumor efficacy of CDDP. ${ }^{28-31}$ Further research is needed to reliably exclude an impact of STS on antitumor activity.

Limitations of the present study are the retrospective nature of the analysis, and the limited number of patients in the analyzed subgroups. Additionally, patients in the STS and no STS group were treated in two successive time intervals introducing potential further bias. However, apart 
from a differing duration of HIPEC due to protocol changes, there were no significant differences in baseline and perioperative characteristics between both groups. Strengths of our study include the highly standardized treatment of patients in our center with a small team of specialized surgeons and defined internal standard operating procedures, as well as the robust results of a multivariate analysis of numerous potential predictive factors for postoperative AKI.

\section{CONCLUSIONS}

In accordance with recent studies, our data strongly suggest that STS effectively protects from CDDP-induced acute renal injury in patients undergoing CRS and HIPEC. Taking into account the controversial data on the benefit of CRS and HIPEC in certain tumor entities and treatment settings, there is an urgent need to prevent complications, such as AKI, which is known to be associated with a prolonged hospital stay and further major morbidities. ${ }^{5}$

Future prospective randomized studies will be needed to validate the benefit of STS among kidney-protective strategies. These studies should also focus on the identification of additional risk factors for AKI following CRS and HIPEC to better define high-risk patients before surgery.

Supplementary Information The online version contains supplementary material available at https://doi.org/10.1245/s10434021-10508-x.

ACKNOWLEDGEMENT The authors thank Anna Lena Rüwald and Keno Lüders for their kind support and the supply of data on the perioperative fluid management of the analyzed patients.

FUNDING Open Access funding enabled and organized by Projekt DEAL.

DISCLOSURE The authors declare no commercial interest in the subject of the study. No entity provided financial or material support.

OPEN ACCESS This article is licensed under a Creative Commons Attribution 4.0 International License, which permits use, sharing, adaptation, distribution and reproduction in any medium or format, as long as you give appropriate credit to the original author(s) and the source, provide a link to the Creative Commons licence, and indicate if changes were made. The images or other third party material in this article are included in the article's Creative Commons licence, unless indicated otherwise in a credit line to the material. If material is not included in the article's Creative Commons licence and your intended use is not permitted by statutory regulation or exceeds the permitted use, you will need to obtain permission directly from the copyright holder. To view a copy of this licence, visit http://creativecommons. org/licenses/by/4.0/.

\section{REFERENCES}

1. Quénet F, Elias D, Roca L, Goéré D, Ghouti L, Pocard M, et al. Cytoreductive surgery plus hyperthermic intraperitoneal chemotherapy versus cytoreductive surgery alone for colorectal peritoneal metastases (PRODIGE 7): a multicentre, randomised, open-label, phase 3 trial. Lancet Oncol. 2021;22:256-66.

2. Hettinga JV, Lemstra W, Meijer C, Dam WA, Uges DR, Konings AW, et al. Mechanism of hyperthermic potentiation of cisplatin action in cisplatin-sensitive and -resistant tumour cells. $\mathrm{Br} \mathrm{J}$ Cancer. 1997;75(12):1735-43.

3. Rossi CR, Mocellin S, Pilati P, Foletto M, Quintieri L, Palatini P, et al. Pharmacokinetics of intraperitoneal cisplatin and doxorubicin. Surg Oncol Clin N Am. 2003;12(3):781-94.

4. Cata JP, Zavala AM, Van Meter A, Williams UU, Soliz J, Hernandez $M$, et al. Identification of risk factors associated with postoperative acute kidney injury after cytoreductive surgery with hyperthermic intraperitoneal chemotherapy: a retrospective study. Int J Hyperther. 2018;34(5):538-44.

5. Naffouje SA, Tulla KA, Chorley R, Armstrong N, Salti GI. Acute kidney injury increases the rate of major morbidities in cytoreductive surgery and HIPEC. Ann Med Surg (Lond). 2018;35:163-8.

6. Sin EI, Chia CS, Tan GH, Soo KC, Teo MC. Acute kidney injury in ovarian cancer patients undergoing cytoreductive surgery and hyperthermic intra-peritoneal chemotherapy. Int $J$ Hyperth. 2017;33(6):690-5.

7. Hakeam HA, Breakiet M, Azzam A, Nadeem A, Amin T. The incidence of cisplatin nephrotoxicity post hyperthermic intraperitoneal chemotherapy (HIPEC) and cytoreductive surgery. Ren Fail. 2014;36(10):1486-91.

8. Bakrin N, Bereder JM, Decullier E, Classe JM, Msika S, Lorimier $\mathrm{G}$, et al. Peritoneal carcinomatosis treated with cytoreductive surgery and hyperthermic intraperitoneal chemotherapy (HIPEC) for advanced ovarian carcinoma: a French multicentre retrospective cohort study of 566 patients. Eur J Surg Oncol. 2013;39(12):1435-43.

9. Brock PR, Maibach R, Childs M, Rajput K, Roebuck D, Sullivan MJ, et al. Sodium thiosulfate for protection from cisplatin-induced hearing loss. N Engl J Med. 2018;378(25):2376-85.

10. Freyer DR, Chen L, Krailo MD, Knight K, Villaluna D, Bliss B, et al. Effects of sodium thiosulfate versus observation on development of cisplatin-induced hearing loss in children with cancer (ACCL0431): a multicentre, randomised, controlled, open-label, phase 3 trial. Lancet Oncol. 2017;18(1):63-74.

11. Pfeifle CE, Howell SB, Felthouse RD, Woliver TB, Andrews PA, Markman M, et al. High-dose cisplatin with sodium thiosulfate protection. J Clin Oncol. 1985;3(2):237-44.

12. Van Driel WJ, Koole SN, Sikorska K, van Leeuwen JHS, Schreuder HW, Hermans RH, et al. Hyperthermic intraperitoneal chemotherapy in ovarian cancer. $N$ Engl J Med. 2018;378(3):230-40.

13. Guastalla JP, Vermorken JB, Wils JA, George M, Scotto V, Nooij $\mathrm{M}$, et al. Phase II trial for intraperitoneal cisplatin plus intravenous sodium thiosulphate in advanced ovarian carcinoma patients with minimal residual disease after cisplatin-based chemotherapy - a phase II study of the EORTC Gynaecological Cancer Cooperative Group. Eur J Cancer. 1994;30A(1):45-9.

14. Van Rijswijk RE, Hoekman K, Burger CW, Verheijen RH, Vermorken JB. Experience with intraperitoneal cisplatin and etoposide and i.v. sodium thiosulphate protection in ovarian cancer patients with either pathologically complete response or minimal residual disease. Ann Oncol. 1997;8(12):1235-41.

15. Laplace N, Kepenekian V, Friggeri A, Vassal O, Ranchon F, Rioufol C, et al. Sodium thiosulfate protects from renal 
impairement following hyperthermic intraperitoneal chemotherapy (HIPEC) with Cisplatin. Int $J$ Hyperther. 2020;37(1):897-902.

16. Mehta RL, Kellum JA, Shah SV, Molitoris BA, Ronco C, Warnock DG, et al. Acute kidney injury network: report of an initiative to improve outcomes in acute kidney injury. Crit Care. 2007;11(2):R31.

17. Jacquet $\mathrm{P}$, Sugarbaker $\mathrm{PH}$. Clinical research methodologies in diagnosis and staging of patients with peritoneal carcinomatosis. Cancer Treat Res. 1996;82:359-74.

18. Sugarbaker PH. Management of peritoneal-surface malignancy: the surgeon's role. Langenbecks Arch Surg. 1999;384(6):576-87.

19. Dindo D, Demartines N, Clavien PA. Classification of surgical complications: a new proposal with evaluation in a cohort of 6336 patients and results of a survey. Ann Surg. 2004;240(2):205-13.

20. Brandl A, Zielinski CB, Raue W, Pratschke J, Rau B. Peritoneal metastases of rare carcinomas treated with cytoreductive surgery and HIPEC - a single center case series. Ann Med Surg. 2012;2017(22):7-11.

21. Verwaal VJ, van Ruth S, de Bree E, van Slooten GW, van Tinteren $\mathrm{H}$, Boot $\mathrm{H}$, et al. Randomized trial of cytoreduction and hyperthermic intraperitoneal chemotherapy versus systemic chemotherapy and palliative surgery in patients with peritoneal carcinomatosis of colorectal cancer. $J$ Clin Oncol. 2003;21(20):3737-43.

22. Tilleman TR, Richards WG, Zellos L, Johnson BE, Jaklitsch MT, Mueller J, et al. Extrapleural pneumonectomy followed by intracavitary intraoperative hyperthermic cisplatin with pharmacologic cytoprotection for treatment of malignant pleural mesothelioma: a phase II prospective study. J Thorac Cardiovasc Surg. 2009;138(2):405-11.

23. Hübner M, Kusamura S, Villeneuve L, Al-Niaimi A, Alyami M, Balonov K, et al. Guidelines for Perioperative Care in Cytoreductive Surgery (CRS) with or without hyperthermic IntraPEritoneal chemotherapy (HIPEC): Enhanced recovery after surgery $($ ERAS $\AA)$ Society Recommendations-Part I: preoperative and intraoperative management. Eur J Surg Oncol. 2020;46(12):2292-310.

24. Hübner M, Kusamura S, Villeneuve L, Al-Niaimi A, Alyami M, Balonov K, et al. Guidelines for perioperative care in cytoreductive surgery (CRS) with or without hyperthermic
IntraPEritoneal chemotherapy (HIPEC): enhanced recovery after surgery $\left(\right.$ ERAS $\left.{ }^{\circledR}\right)$ Society recommendations-Part II: postoperative management and special considerations. Eur J Surg Oncol. 2020;46(12):2311-23.

25. Lu PW, Fields AC, Shabat G, Bleday R, Goldberg JE, Irani J, et al. Cytoreductive surgery and HIPEC in an enhanced recovery after surgery program: a feasibility study. J Surg Res. 2020;247:59-65.

26. Martin RC 2nd, Marshall BM, Philips P, Egger M, McMasters $\mathrm{KM}$, Scoggins CR. Enhanced recovery after surgery is safe for cytoreductive surgery with hyperthermic intraperitoneal chemotherapy. Am J Surg. 2020;220(6):1428-32.

27. Webb C, Day R, Velazco CS, Pockaj BA, Gray RJ, Stucky CC, et al. Implementation of an enhanced recovery after surgery (ERAS) program is associated with improved outcomes in patients undergoing cytoreductive surgery and hyperthermic intraperitoneal chemotherapy. Ann Surg Oncol. 2020;27(1):303-12.

28. Harned TM, Kalous O, Neuwelt A, Loera J, Ji L, Iovine P, et al. Sodium thiosulfate administered six hours after cisplatin does not compromise antineuroblastoma activity. Clin Cancer Res. 2008;14(2):533-40.

29. Inoue M, Shimizu C, Shimizu H, Tanizawa O. Neutralizing effect of sodium thiosulfate on antitumor efficacy of cisplatin for human carcinoma xenografts in nude mice. Gynecol Oncol. 1991;40(1):34-7.

30. Saito D, Fujii T, Yoshida S, Ohkura H, Oguro Y. Effect of sodium thiosulfate on the antitumor effect and nephrotoxicity of CDDP in human gastric cancer transplanted in nude mice. Gan To Kagaku Ryoho. 1989;16(3 Pt 1):379-85.

31. Muldoon LL, Pagel MA, Kroll RA, Brummett RE, Doolittle ND, Zuhowski EG, et al. Delayed administration of sodium thiosulfate in animal models reduces platinum ototoxicity without reduction of antitumor activity. Clin Cancer Res. 2000;6(1):309-15.

32. Doyle DJ, Goyal A, Bansal P, Garmon EH, American Society of Anesthesiologists Classification, in StatPearls. 2020. Treasure Island (FL).

Publisher's Note Springer Nature remains neutral with regard to jurisdictional claims in published maps and institutional affiliations. 